\title{
Challenges to primary care from co- and multi-morbidity
}

Medical education is based largely on the challenges of providing high-quality care for specific diseases. Most learning in medical school is carried out by teacher-researchers with expertise in one particular disease or, at best, teachers with special knowledge in one type of disease or an intervention to treat or manage one type of health problem, leading to health care in many countries being led by specialist rather than generalist medicine. Increased effectiveness of health services' interventions that delay death by managing (although not necessarily curing) diseases has led to a marked increase in the coexistence of separate diseases in individual people. Older literature expressed this notion by the term 'co-morbidity': the co-occurrence of unrelated diseases. Total morbidity is not the same as the sum of different diseases - despite the fact that virtually all population data on diseases assumes that it is. The sum of deaths attributed to individual diseases in the world is greater than the total number of deaths (Murray et al., 2004).

Neither morbidity nor multi-morbidity is randomly distributed in populations. People and populations differ in their overall vulnerability to illness and resistance to threats to their health; some have more than their share of illness and some have less. Clustering of diseases is a result of a complex pattern of interacting influences, extending far beyond biological vulnerability. It is more common in socially deprived populations and more common in children as compared with its expected frequency based on frequency of individual diseases in populations (despite lower frequencies of morbidity). This morbidity mix (sometimes called 'case-mix' by health services managers) is often called multi-morbidity. When considered in the context of demands on health services, it is known as 'morbidity burden'.

Over time, and particularly in the last decade or two, the frequency of diagnosed morbidity has increased, at least partly as a result of lowered thresholds for diagnosis, inclusion of new diagnoses (including some risk factors, such as obesity) and perhaps also as a result of true increases in some

(C) Cambridge University Press 2011 diseases (such as those resulting from environmental insults over time). As a result of these changes, the frequency of multi-morbidity is increasing. It is not necessarily the case that increased multi-morbidity would be associated with increased morbidity burden. For example, among the elderly in the United States, the percentage of people with five or more diagnosed conditions who reported being in excellent or good health increased from $10 \%$ to $30 \%$ between 1987 and 2002 (Thorpe and Howard, 2006). Thus, morbidity has decreased (by self-reports), but physicians are generating more interventions for the diagnosed conditions and, hence, greater burden on the health system.

Increases in multi-morbidity are associated with great increases in costs of care, hospitalizations that should be preventable, and adverse events (Wolff et al., 2002). Because it is also associated with increased likelihood of referrals (Forrest et al., 2006), it has great impact on the balance of use of services between primary care and specialist physicians. Although disease-oriented specialists tend to see people with LESS severe disease (Hartz and James, 2006), they dominate the care of people with high burdens of morbidity because of the multiplicity of disease types and, therefore, different types of specialists; this is especially the case in the elderly, at least in the United States (Starfield et al., 2003). Because excessive use of specialist care is unnecessary, potentially dangerous, and very costly (Starfield et al., 2005), consideration of their appropriate use is warranted.

Use of specialist services varies widely from place to place, even after controlling for degree of morbidity; in the United Kingdom, it is approximately one-third of that among insured people in health plans in the United States (Forrest et al., 2002). Although much higher than in the United Kingdom, it is lower in both Canada and Spain than in the United States. This is the case both for total number of specialist visits as well as proportion of the population with at least one specialist visit in any given year. Controlled for degree of multi-morbidity, the more different 
generalists who are seen, the more DIFFERENT specialists are seen, especially in people with high morbidity burdens, independent of the number of visits to generalists. The more different specialists seen, the higher the total costs, medical costs, diagnostic tests and interventions, and types of medication prescribed (Starfield et al., 2009).

Studies in different countries have shown that it is not chronic conditions by themselves that raise resource use. Rather, it is the number of types of conditions, that is, multi-morbidity. In British Columbia (Canada), people with acute conditions only, with any chronic condition, or with high impact chronic conditions have the SAME resource use after stratifying for morbidity burden; only increasing morbidity burden is associated with increased resource use (Broemeling et al., 2005). In Ontario (Canada), degree of morbidity burden is the most salient influence (among self-rated health, disability, number of chronic conditions, and age $\geq 65$ years) on both visits to primary care physicians and specialists (Sibley et al., 2010). In Israel, multimorbidity is the most salient influences on costs of care - much more important as influences than are age, gender, or a chronic condition count or index (Shadmi et al., 2010 ).

Thus, despite the attention to chronic diseases in many countries of the world, it is not chronicity per se that creates a burden on the health-care system. The reason for this is that many non-chronic conditions behave as if they were chronic-persisting or recurring over time. Conversely, chronic illnesses are associated with and predispose to acute illnesses.

In conclusion, we know that multi-morbidity is increasing and that specialist use is increasing. We also know that it is not 'chronic diseases' by themselves that are the challenge. What we do not know is the appropriate role of specialists (and hence, referrals) in the management of people with multi-morbidity. Because of changing health needs, disease-oriented care must be replaced by personoriented care over time. That is, we need more and better primary care.

\section{Barbara Starfield Health Policy and Management Johns Hopkins University Baltimore, MD, USA Email: bstarfie@jhsph.edu}

\section{References}

Broemeling, A., Watson, D. and Black, C. 2005: Chronic conditions and co-morbidity among residents of British Columbia. Vancouver, BC: University of British Columbia.

Forrest, C.B., Majeed, A., Weiner, J.P., Carroll, K. and Bindman, A.B. 2002: Comparison of specialty referral rates in the United Kingdom and the United States: retrospective cohort analysis. British Medical Journal 325, 370-71.

Forrest, C.B., Nutting, P.A., von Schrader, S., Rohde, C. and Starfield, B. 2006: Primary care physician specialty referral decision making: patient, physician, and health care system determinants. Medical Decision Making 26, 76-85.

Hartz, A. and James, P.A. 2006: A systematic review of studies comparing myocardial infarction mortality for generalists and specialists: lessons for research and health policy. Journal of the American Board of Family Medicine 19, 291-302.

Murray, C.J., Lopez, A.D. and Wibulpolprasert, S. 2004: Monitoring global health: time for new solutions. British Medical Journal 329, 1096-100.

Shadmi, E., Balicer, R.D., Kinder, K., Abrams, C., Starfield, B., and Weiner, J.P. 2010: Morbidity and health care resource use: beyond chronic condition counts (submitted for publication).

Sibley, L.M., Moineddin, R., Agha, M.M. and Glazier, R.H. 2010: Risk adjustment using administrative data-based and survey-derived methods for explaining physician utilization. Medical Care 48, 175-82.

Starfield, B., Lemke, K.W., Bernhardt, T., Foldes, S.S., Forrest, C.B. and Weiner, J.P. 2003: Comorbidity: implications for the importance of primary care in 'case' management. Annals of Family Medicine 1, 8-14.

Starfield, B., Shi, L., Grover, A. and Macinko, J. 2005: The effects of specialist supply on populations' health: assessing the evidence. Health Affairs W5, 97-107.

Starfield, B., Chang, H., Lemke, K.W. and Weiner, J.P. 2009: Ambulatory specialist use by non-hospitalized patients in US health plans: correlates and consequences. Journal of Ambulatory Care Management 32, 216-25.

Thorpe, K.E. and Howard, D.H. 2006: The rise in spending among Medicare beneficiaries: the role of chronic disease prevalence and changes in treatment intensity. Health Affairs 25, W378-88.

Wolff, J.L., Starfield, B. and Anderson, G. 2002: Prevalence, expenditures, and complications of multiple chronic conditions in the elderly. Archives of Internal Medicine 162, 2269-76. 